\title{
MiR-5195-3p functions as a tumor suppressor in prostate cancer via targeting CCNL1
}

Xing Zeng, Zhiquan Hu, Yuanqing Shen, Xian Wei, Jiahua Gan and Zheng Liu*

\section{${ }^{*}$ Correspondence:}

liu_zheng0205@126.com Department of Urology,

Tongji Hospital, Tongji

Medical College, Huazhong

University of Science

and Technology, No. 1095

Jiefang Ave, Wuhan 430030,

Hubei, China

\begin{abstract}
Background: Accumulating evidence indicates that miR-5195-3p exerts tumorsuppressive roles in several tumors. However, the clinical significance and biological function of miR-5195-3p in prostate cancer (PCa) have not been reported yet.
\end{abstract}

Methods: The expression levels of miR-5195-3p and Cyclin L1 (CCNL1) were determined using quantitative real-time PCR in clinical specimens and cell lines. The clinical significance of miR-5195-3p in patients with PCa was evaluated using Kaplan-Meier survival analysis and Cox regression models. Cell proliferation and cell cycle distribution were measured by CCK-8 assay and flow cytometry, respectively. The association between miR-5195-3p and CCNL1 was analyzed by luciferase reporter assay.

Results: MiR-5195-3p expression levels were significantly downregulated in 69 paired PCa tissues compared with matched adjacent normal tissues. The decreased miR5195-3p expression was associated with Gleason score and TNM stage, as well as worse survival prognosis. The in vitro experiments showed that miR-5195-3p overexpression suppressed the proliferation and cell cycle G1/S transition in PC-3 and DU145 cells. Elevated miR-5195-3p abundance obviously impaired tumor formation in vivo using PC-3 xenografts. Mechanistically, CCNL1 was a direct target of miR-5195-3p in PCa cells, which was inversely correlated with miR-5195-3p in PCa tissues. Importantly, CCNL1 knockdown imitated, while overexpression reversed, the effects of miR-5195-3p overexpression on PCa cell proliferation and cell cycle G1/S transition.

Conclusions: Our data suggest that miR-5195-3p functions as a tumor suppressor by targeting CCNL1 in PCa.

Keywords: Prostate cancer, miR-5195-3p, CCNL1, Proliferation

\section{Background}

Prostate cancer ( $\mathrm{PCa})$, as the most frequently diagnosed male malignancy, has been the leading cause of tumor-related deaths worldwide, with its pathological and clinical heterogeneity [1,2]. It is estimated that there are more than 220,000 new cases of PCa and over 30,000 death per year in the USA [3]. Despite great improvement in the outcomes of PCa at early stage by early prostate-specific antigen (PSA) testing, surgical resection, and androgen deprivation therapy $[4,5]$, the prognosis is still poor for patients at advanced stage, especially for those with emergence of castration-resistant PCa [6]. Therefore,

(c) The Author(s) 2022. Open Access This article is licensed under a Creative Commons Attribution 4.0 International License, which permits use, sharing, adaptation, distribution and reproduction in any medium or format, as long as you give appropriate credit to the original author(s) and the source, provide a link to the Creative Commons licence, and indicate if changes were made. The images or other third party material in this article are included in the article's Creative Commons licence, unless indicated otherwise in a credit line to the material. If material is not included in the article's Creative Commons licence and your intended use is not permitted by statutory regulation or exceeds the permitted use, you will need to obtain permission directly from the copyright holder. To view a copy of this licence, visit http:// creativecommons.org/licenses/by/4.0/. 
elucidation of the molecular mechanisms underlying the initiation and progression of PCa is urgently needed to establish new therapeutic targets for PCa treatment.

MicroRNAs (miRNAs/miRs) are a group of small, noncoding, and single-stranded RNAs approximately 18-22 nucleotides in length, which can regulate multiple physiological processes by selectively inhibiting the downstream target mRNAs via binding to their $3^{\prime}$-untranslated region ( $3^{\prime}$-UTR) $[7,8]$. In recent years, growing evidence has suggested that certain aberrantly expressed miRNAs can act as either oncogenes or tumor suppressors, thereby affecting the pathogenesis of PCa. For example, overexpression of $m i R-139$ inhibited the cell growth and migration in PCa cells [9]. Overexpression of $m i R-589-5 p$ inhibited cell viability, migration, and invasion in PCa cells [10]. On the contrary, some oncogenic factors, including miR-410-3p [11], miR-153 [12], and miR191 [13], have been identified as carcinogenic factors that predict poor prognosis and promote the proliferation of PCa cells. Notably, miR-5195-3p, a relatively poorly studied miRNA, has been shown to participate in cell biological processes that regulate the progression of tumors, including non-small cell lung cancer [14], glioma [15], osteosarcoma [16], and bladder cancer [17]. However, there are limited studies on the clinical significance and biological function of $m i R-5195-3 p$ in PCa.

Cyclin L1 (CCNL1 also termed Ania-6a), localized in the chromosomal 3q25 region, codes for a putative key regulator of pre-mRNA processing and is involved in G1/S transition during the cell cycle [18]. Interestingly, CCNL1 has been illustrated as a potential target of therapeutic interventions implicated in carcinogenesis [19]. As reported by Redon et al. [20] and Sticht et al. [21], CCNL1 was overexpressed and amplified in human head and neck squamous cell carcinoma. Moreover, CCNL1 has been demonstrated to be a direct target gene of miR-199b-5p and be involved in miR-199b-5p suppressing cell proliferation and inducing cell cycle arrest and cell apoptosis in Ewing's sarcoma [22]. According to the online software program TargetScan 7.1 prediction that CCNL1 was a potential target of miR-5195-3p, we speculated that miR-5195-3p might play an important role in PCa tumorigenesis by targeting CCNL1 via affecting cell cycle progression.

Therefore, we first investigated the expression pattern and clinical significance of miR$5195-3 p$ in PCa tissues. Next, we conducted a series of in vitro and in vivo functional experiments to observe the regulatory roles of $m i R-5195-3 p$ in cell proliferation and tumor growth. Furthermore, we validated the association between miR-5195-3p and CCNL1 in PCa. This research will enhance our understanding of PCa biology and provide new insights into molecular therapy for PCa treatment.

\section{Materials and methods}

\section{Patients and tissue samples}

In total, 69 pairs of tumor tissues and matched adjacent normal tissues were obtained from patients with $\mathrm{PCa}$ who underwent radical prostatectomy at Tongji Hospital, Tongji Medical College, Huazhong University of Science and Technology (Hubei, China) between March 2016 and September 2018. Prior to radical prostatectomy, all patients were confirmed not to receive chemotherapy, radiotherapy, or androgen deprivation therapies. All tissue specimens were immediately stored at $-80{ }^{\circ} \mathrm{C}$ until further use. The basic clinicopathological characteristics of all patients with $\mathrm{PCa}$, including age, 
Gleason score, and TNM stage, are summarized in Table 1. All enrolled patients underwent 5-year follow-up through telephone. Written informed consent was signed by all patients, and the present study was approved by the ethics committee of Tongji Hospital, Tongji Medical College, Huazhong University of Science and Technology (approval number TMCU-87DG; 2016.3.12; Hubei, China).

\section{Cell culture}

Human PCa cell lines (PC-3, 22RV1, DU145, and LNCaP) and a nontransformed but immortalized prostate cell line RWPE-1 were purchased from the American Type Culture Collection (Manassas, VA, USA). All cell lines were cultured in RPMI-1640 (Gibco; Thermo Fisher Scientific) supplemented with $10 \%$ fetal bovine serum (Gibco) in a humidified incubator containing $5 \% \mathrm{CO}_{2}$ at $37^{\circ} \mathrm{C}$.

\section{Cell transfection}

MiR-5195-3p mimics, scrambled miRNA (miR-NC), smaller interfering RNA against CCNL1 (si-CCNL1), negative control (si-NC), the overexpression plasmid of pcDNA3.1CCNL1, and empty vector pcDNA3.1 were synthesized from Shanghai GenePharma. For cell transfection, PC-3 and DU145 cells were seeded into six-well plates and transfected with the above oligonucleotides using Lipofectamine 2000 (Thermo Fisher Scientific) according to the manufacturer's instructions.

\section{Quantitative real-time PCR}

Total RNA was extracted using mirVana miRNA isolation kit (Life Technologies; Thermo Fisher Scientific) for miRNA and RNeasy mini kit (Qiagen, Valencia, CA, USA) for mRNA. The synthesis of complementary DNA was performed using miScript II RT kit (Applied Biosystems, CA) for miRNA and superscript VILO cDNA kit (Thermo

Table 1 Relationship between miR-5195-3p expression and clinicopathological characteristics of patients with prostate cancer

\begin{tabular}{|c|c|c|c|c|}
\hline \multirow[t]{2}{*}{ Characteristic } & \multirow{2}{*}{$\begin{array}{l}\text { Case } \\
(n=69)\end{array}$} & \multicolumn{2}{|c|}{ miR-5195-3p expression } & \multirow{2}{*}{$\begin{array}{l}p \text {-Value } \\
\text { (chi-squared test) }\end{array}$} \\
\hline & & Low $(n=35)$ & High $(n=34)$ & \\
\hline Age (year) & & & & 0.733 \\
\hline$<65$ & 21 & 10 & 11 & \\
\hline$\geq 65$ & 48 & 25 & 23 & \\
\hline Preoperative PSA (ng/ml) & & & & 0.368 \\
\hline$<10$ & 43 & 20 & 23 & \\
\hline$\geq 10$ & 26 & 15 & 11 & \\
\hline Gleason score & & & & $0.001^{*}$ \\
\hline$<7$ & 51 & 20 & 31 & \\
\hline$\geq 7$ & 18 & 15 & 3 & \\
\hline TNM stage & & & & $0.006^{*}$ \\
\hline$|/| \mid$ & 46 & 18 & 28 & \\
\hline III/IV & 23 & 17 & 6 & \\
\hline Metastasis & & & & 0.118 \\
\hline No & 39 & 23 & 16 & \\
\hline Yes & 30 & 12 & 18 & \\
\hline
\end{tabular}


Fisher Scientific) for mRNA according to the manufacturer's instructions. Quantitative real-time PCR was performed using miScript SYBR Green PCR kit (Qiagen) for miR5195-3p or SYBR Green PCR kit (Applied Biosystems, CA) for CCNL1 mRNA levels with the specific primer sequences synthesized by Sangon Biotech (Shanghai). Each experiment was performed in triplicate, and relative abundance was normalized to U6 for miR-5195-3p or GADPH for CCNL1 mRNA by the $2^{-\triangle \Delta C T}$ method. The primer sequences used were as follows: CCNL1, forward 5'-GGAAAAAGGACTCCAAGC CC-3' ${ }^{\prime}$ and reverse 5'-GCTGCAAGGTAGATGCAAGC-3'; GAPDH, forward 5'-GGT GAAGGTCGGAGTCAACG-3' and reverse 5'-GCATCGCCCCACTTGATTTT- ${ }^{\prime}$ '.

\section{Cell proliferation assay}

Transfected PCa cells at a density of $3 \times 10^{4}$ cells per well in technical triplicates were seeded in six-well plates and cultured for 24,48 , and $72 \mathrm{~h}$, respectively. At each timepoint, cells in each well were incubated with $10 \mu$ of CCK-8 solution (Sigma-Aldrich) for $2 \mathrm{~h}$. Afterwards, the absorbance at a wavelength of $450 \mathrm{~nm}$ was measured in each well using a microplate reader.

\section{Cell cycle analysis}

Transfected PCa cells at a density $4 \times 10^{5}$ cells per well were seeded in six-well plates and incubated for $48 \mathrm{~h}$. Subsequently, cells were washed with PBS and fixed with cold 70\% ethanol overnight, followed by incubation with $0.1 \mathrm{mg} / \mathrm{ml}$ propidium iodide (SigmaAldrich) for $30 \mathrm{~min}$ in the dark. Next, the cells were analyzed by flow cytometry (BD Biosciences, Franklin Lakes, NJ, USA) with FlowJo software (Version 10.0.4; FlowJo LLC).

\section{Luciferase reporter assay}

According to the putative binding sites predicted for miR-5195-3p with the $3^{\prime}$-UTR of CCNL1 by the online software program TargetScan 7.1 (http://www.targetscan.org), we performed luciferase reporter assay to validate the above prediction. In brief, the fragments of CCNL1 3'-UTR containing either putative miR-5195-3p seed sequence or corresponding mutant (MUT) sites using QuickChange Site-Direct Mutagenesis Kit (Stratagene) were subcloned into psiCHECK-2 vector (Promega, USA) to obtain the reporter plasmids of CCNL1-wild type (WT) and CCNL1-MUT, respectively. Then, PC-3 or DU145 cells were plated in 24-well plates and co-transfected with $1 \mu \mathrm{g}$ reporter plasmid CCNL1-WT or CCNL1-MUT together with $30 \mathrm{nM}$ miR-5195-3p mimics or miR-NC for $48 \mathrm{~h}$. Relative luciferase activity was determined using a Dual-Luciferase Reporter Assay System (Promega).

\section{Tumor xenograft formation}

For the tumorigenicity assay, miR-NC or miR-5195-3p mimics stably transfected overexpression $1.8 \times 10^{6} \mathrm{PC}-3$ cell suspension was injected subcutaneously into the right flank of 4-5-week-old BALB/c nude mice (Shanghai Laboratory Animal Research Center, Shanghai, China) with five mice in each group. Mice were monitored every 5 days, and the tumor length/width was measured using calipers. Tumor volume was calculated using the modified ellipsoid formula: volume $=1 / 2\left(\right.$ length $\times$ width $\left.^{2}\right)$. At the 
end of 30 days, all the mice were killed by cervical dislocation. Then, the tumor weight was measured and tumor tissues were harvested for analyzing the expression levels of miR-5195-3p, as well as protein levels of Cyclin L1, CDK4, and Cyclin D1. All animal experiments were performed in accordance with the Huazhong University of Science and Technology Research Institute Animal Care Committee guidelines (approval number HUST-58A; 2017.6.23; Hubei, China).

\section{Western blot analysis}

Total protein samples were extracted from cell lines or tumor tissues with RIPA lysis buffer (Thermo Fisher Scientific), and protein concentration was analyzed using a BCA Protein Assay Kit (Pierce, Rockford, IL, USA) according to the manufacturer's instructions. Then, equal amount of protein sample $(30 \mu \mathrm{g})$ was subjected to electrophoresis using sodium dodecyl sulfate polyacrylamide gels (SDS-PAGE), which was subsequently transferred onto PVDF membranes. Next, the membranes were blocked with 5\% nonfat dried milk in TBST for $2 \mathrm{~h}$ and incubated with primary antibodies against Cyclin L1 (1:1000, no. PA5-36070, Thermo Fisher Scientific), CDK4 (1:1000, ab226474, Abcam), Cyclin D1 (1:5000, ab226977, Abcam), and GAPDH (1:1,000; ab37168, Abcam) overnight at $4{ }^{\circ} \mathrm{C}$. Subsequently, the membranes were incubated with horseradish-peroxidase-linked secondary antibodies (1:5000; cat. no. SC-2054; Santa Cruz Biotechnology) for $2 \mathrm{~h}$ at room temperature. Finally, the protein bands were visualized by a chemiluminescence detection kit (ECL, Millipore, USA).

\section{Statistical analysis}

The GraphPad Prism 6.0 software (National Institutes of Health, Bethesda, MD, USA) was used to perform all statistical analysis. The association between miR-5195-3p expression and PCa clinicopathologic characteristics was assessed by chi-squared test. The Kaplan-Meier method was used to generate survival curves. Univariate and multivariate Cox regression models were constructed to estimate the hazard ratios (HRs) of independent factors affecting the overall survival in patients with PCa. The Spearman's correlation coefficient was used to analyze the association between miR-5195-3p expression and CCNL1 expression in PCa tissues. All the quantitative data were expressed as mean \pm standard deviation (SD) of at least three experimental replicates. The differences among groups were analyzed using either the one-way ANOVA or the Student's $t$-test. Statistical significance was defined as $p$-value less than 0.05 .

\section{Results}

MiR-5195-3p was downregulated in PCa tissues, which was correlated with cancer progression

We first performed quantitative real-time PCR analysis to determine the expression level of miR-5195-3p in 69 pairs of human PCa and adjacent tissues. As shown in Fig. 1A, miR-5195-3p expression was significantly lower in PCa tissues than in adjacent normal tissues. We then sought to explore the association between miR-5195-3p expression and clinical characteristics. According to the median value of miR-5195-3p expression, 69 patients were classified into low miR-5195-3p expression group $(n=35)$ and high miR-5195-3p expression group $(n=34)$. As listed in Table 1, miR-5195-3p expression 

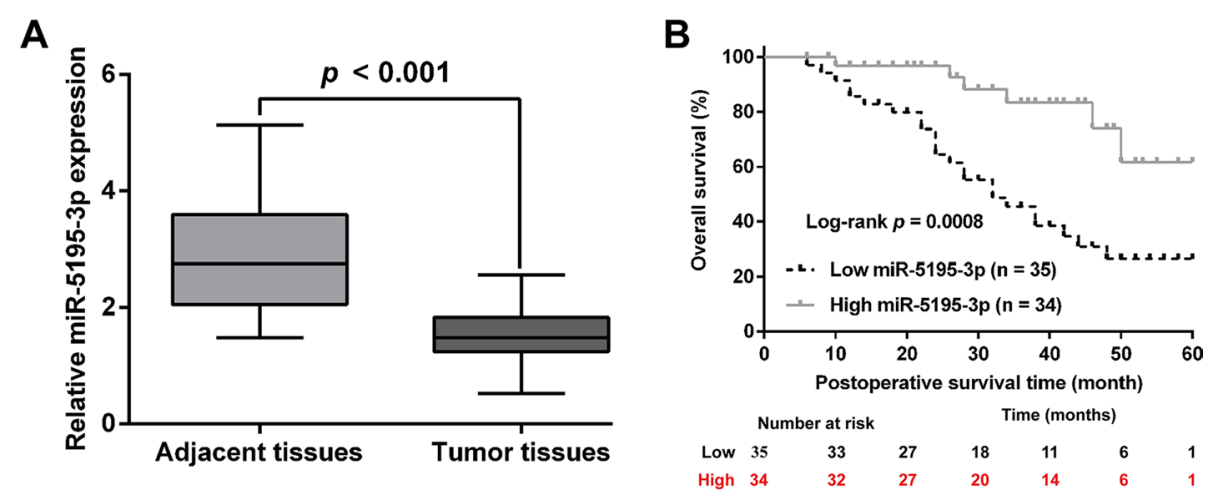

Fig. 1 MiR-5195-3p was downregulated in PCa tissues and correlated with overall survival. A MiR-5195-3p expression in 69 paired HCC and the matched adjacent normal tissue samples was measured by quantitative real-time PCR. B The correlation between miR-5195-3p expression and overall survival was analyzed with the Kaplan-Meier method. The $p$-value was obtained using the log-rank test

Table 2 Univariate and multivariate analysis for overall survival in patients with prostate cancer

\begin{tabular}{|c|c|c|c|c|}
\hline \multirow[t]{2}{*}{ Characteristic } & \multicolumn{2}{|l|}{ Univariate analysis } & \multicolumn{2}{|c|}{ Multivariate analysis } \\
\hline & $\mathrm{HR}(95 \% \mathrm{Cl})$ & $p$ value & $\mathrm{HR}(95 \% \mathrm{Cl})$ & $p$ value \\
\hline Age & $0.895(0.563-1.498)$ & 0.754 & - & - \\
\hline Preoperative PSA (ng/ml) & $2.145(1.284-3.315)$ & 0.415 & - & - \\
\hline Gleason score & $1.432(0.895-2.546)$ & $0.023^{*}$ & $1.365(0.968-2.584)$ & 0.056 \\
\hline TNM stage & $2.312(1.204-2.978)$ & $0.005^{*}$ & $2.542(1.432-3.142)$ & $0.024^{*}$ \\
\hline Metastasis & $3.142(2.142-4.321)$ & 0.064 & - & - \\
\hline MiR-5195-3p expression & $1.759(1.006-2.153)$ & $0.014^{*}$ & $2.132(1.354-2.856)$ & $0.007^{*}$ \\
\hline
\end{tabular}

was significantly associated with Gleason score and TNM stage. We further explored whether miR-5195-3p expression was associated with the prognosis of patients with PCa. Kaplan-Meier survival analysis revealed that patients with PCa with high miR5195-3p expression had longer overall survival than those with low miR-5195-3p expression (Fig. 1B). We also performed univariate and multivariate Cox regression analyses of prognostic indicators using collected clinical specimens (Table 2). Univariate analysis indicated that low miR-5195-3p expression (HR 1.759, $p=0.014$ ), Gleason score $>7$ (HR 1.432, $p=0.023$ ), and TNM stage (HR 2.312, $p=0.005$ ) were independent risk factors for prognosis of patients with PCa. Multivariate analysis suggested that TNM stage (HR 2.542, $p=0.024)$ and low miR-5195-3p expression (HR 2.132, $p=0.007)$ were the hazard factors predicting overall survival in patients with PCa.

\section{MiR-5195-3p overexpression suppressed PCa cell proliferation and cell cycle G1/S transition in vitro}

Subsequently, the expression level of miR-5195-3p was assessed in several PCa cell lines. Consistently, miR-5195-3p expression levels were found to be significantly decreased in PCa cell lines (PC-3, 22RV1, DU145, and LNCaP) compared with a nontransformed but immortalized prostate cell line RWPE-1 (Fig. 2A). To test the biological function 


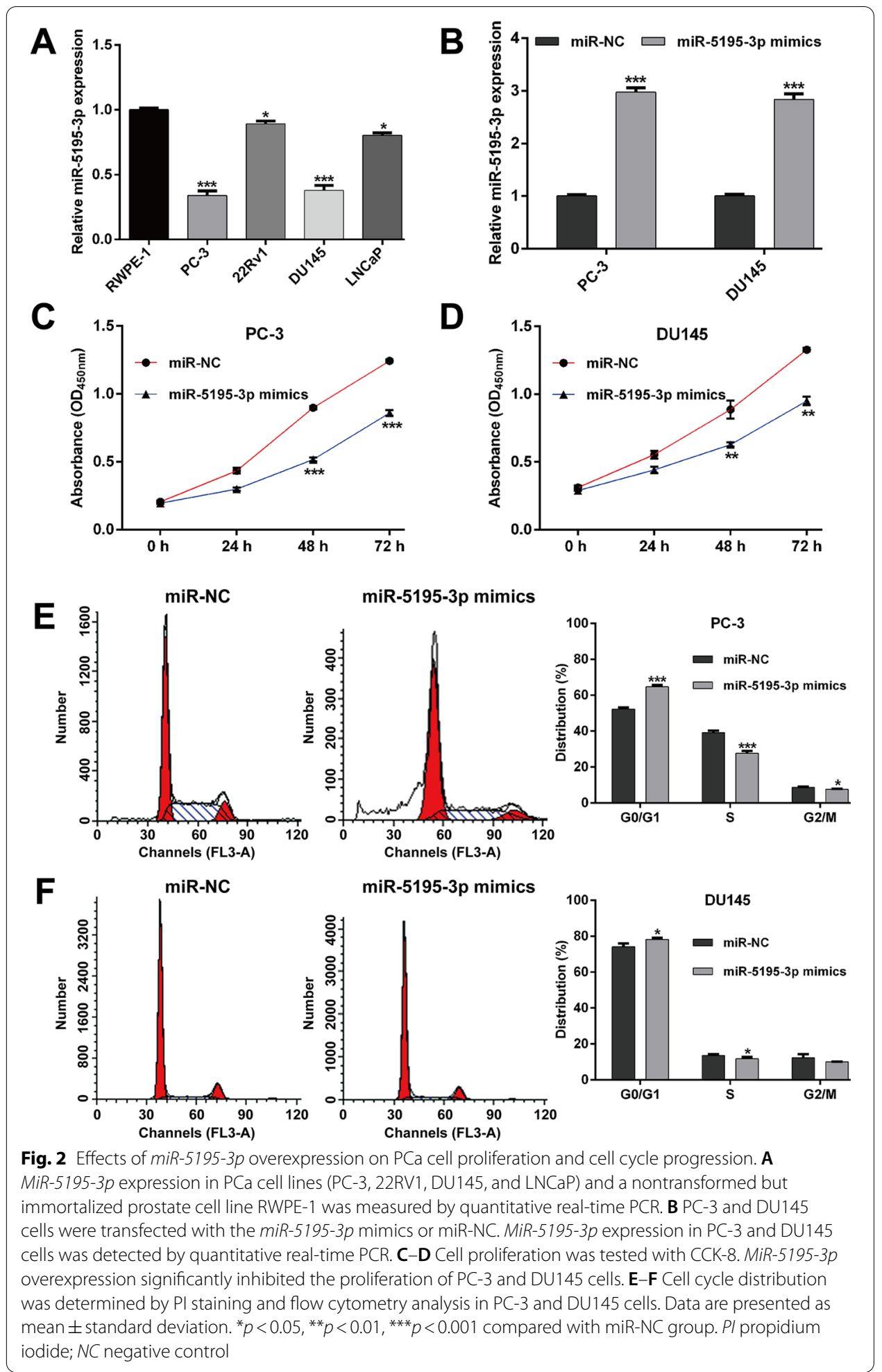

of $m i R-5195-3 p$ in PCa in vitro, PC-3 and DU145 cells were transfected with miR5195-3p mimics or miR-NC. As shown in Fig. 2B, miR-5195-3p expression was significantly elevated in both PC-3 and DU145 cells after miR-5195-3p mimics transfection compared with miR-NC transfection. Next, we performed gain-of-function assays in 
the above constructed miR-5195-3p overexpression cell lines. The results from CCK-8 assay showed that the cell growth curves were remarkably suppressed in PC-3 (Fig. 2C) and DU145 (Fig. 2D) cells after miR-5195-3p overexpression, especially at 48 and $72 \mathrm{~h}$, respectively. Considering uncontrolled cell proliferation was correlated with cell cycle progression, we further analyzed the effects of $m i R-5195-3 p$ overexpression on cell cycle distribution. The results from flow cytometry analysis illustrated that the percentage of cells at G0/G1 phase $(64.71 \% \pm 1.04 \%$ versus $52.27 \% \pm 0.83 \%, \mathrm{p}<0.001)$ was significantly increased, while cells at $\mathrm{S}$ phase $(27.68 \% \pm 1.32 \%$ versus $39.09 \% \pm 1.17 \%, \mathrm{p}<0.001)$ and $\mathrm{G} 2 / \mathrm{M}$ phase $(7.61 \% \pm 0.28 \%$ versus $8.64 \% \pm 0.34 \%, p<0.05)$ were decreased in $m i R$ $5195-3 p$ mimics group compared with miR-NC group in PC-3 cells (Fig. 2E). Similarly, we observed that miR-5195-3p overexpression caused a significant increase in the proportion of cells at G0/G1 phase and decrease in cells at S phase in DU145 cells (Fig. 2F). These findings indicated that $m i R-5195-3 p$ overexpression inhibited the proliferation and induced G0/G1 arrest in PCa cells.

\section{CCNL1 is a direct target of miR-5195-3p in PCa}

To identify the potential effectors of $m i R-5195-3 p$ in PCa progression, the target genes of $m i R-5195-3 p$ were searched by performing bioinformatics analysis. We found that CCNL1 3'-UTR contains one miR-5195-3p-binding site and then constructed vectors containing the WT or MUT $3^{\prime}$-UTR of human CCNL1 fused downstream of the firefly luciferase gene (Fig. 3A). The results from luciferase reporter assay showed that the luciferase activity was significantly decreased in both PC-3 (Fig. 3B) and DU145 (Fig. 3C) cells after co-transfection of miR-5195-3p mimics with CCNL1-WT reporters, which was abolished by the mutations in the putative $m i R-5195-3 p$ binding site. Quantitative real-time PCR (Fig. 3D) and western blot analysis (Fig. 3E) further demonstrated that $C C N L$ mRNA and protein expression levels were both significantly suppressed after miR-5195-3p overexpression in PC-3 and DU145 cells. In addition, the quantitative realtime PCR results showed that CCNL1 mRNA expression was remarkably upregulated in PCa tissues compared with that in matched adjacent normal tissues (Fig. 3F), which was inversely correlated with miR-5195-3p expression (Fig. 3G, $r=-0.2387, p=0.0483$ ). Collectively, these results suggest that $m i R-5195-3 p$ directly targeted CCNL1 in PCa cells to downregulate $C C N L 1$ expression.

MiR-5195-3p suppressed cell proliferation and cell cycle G1/S transition by targeting CCNL1 To confirm whether CCNL1 was the important downstream mediator involved in miR5195-3p regulating PCa cell proliferation and cell cycle progression, we performed lossof-function assays by transfection with si-CCNL1 or si-NC and rescue experiments by co-transfection with miR-5195-3p mimics and pcDNA3.1-CCNL1 in PC-3 cells. As shown in Fig. 4A, the protein expression of Cyclin L1 was obviously suppressed by siCCNL1 transfection, which was recovered by pcDNA3.1-CCNL1 transfection in PC-3 cells. Subsequently, CCK-8 assay revealed that CCNL1 knockdown suppressed, while overexpression promoted, the PC-3 cell proliferation (Fig. 4B). Furthermore, we found that CCNL1 knockdown imitated (Fig. 4C), while overexpression (Fig. 4D) reversed, the effects of $m i R-5195-3 p$ overexpression on cell cycle G1/S transition. These results 

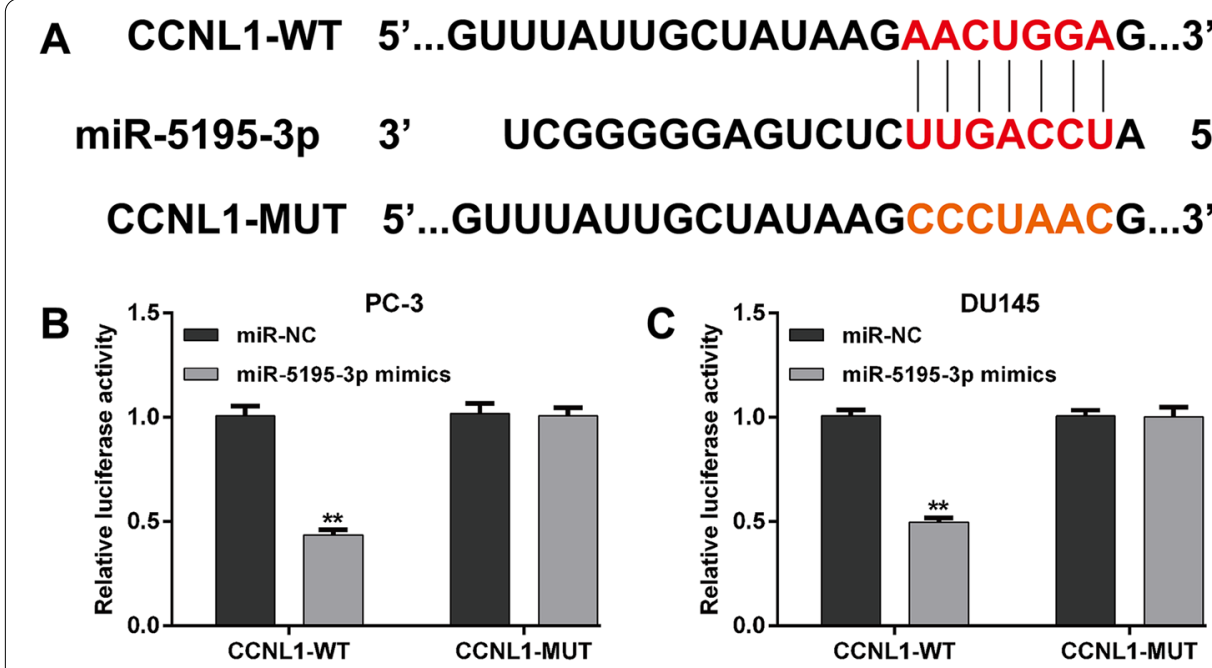

D
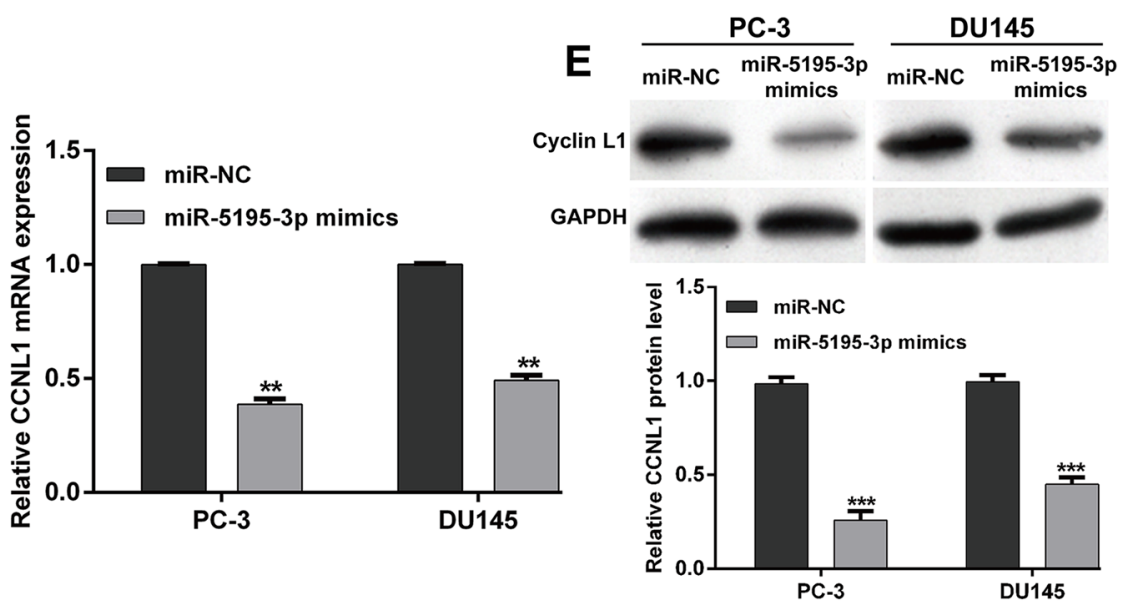

$\mathbf{F}$

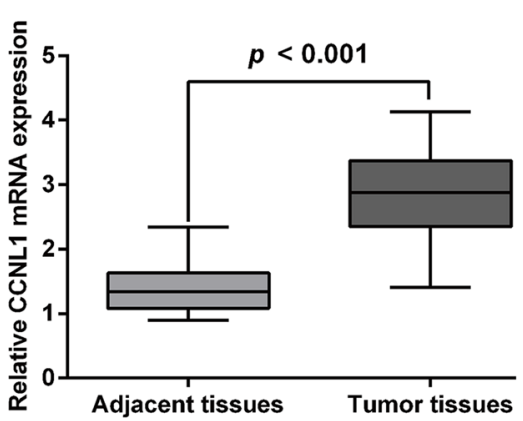

$\mathbf{G}$

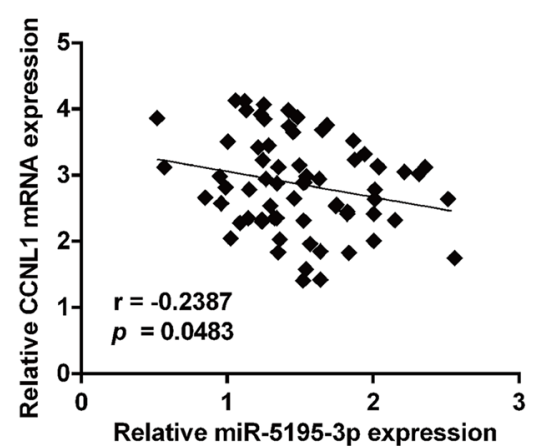

Fig. 3 CCNL1 was a direct target of miR-5195-3p in PCa. A The sequences of the putative miR-5195-3p binding sites in the wild-type and mutant CCNL1 3'-UTR. B-C Luciferase reporter plasmids carrying the CCNL1 wild-type 3'-UTR (CCNL1-WT) or CCNL1 mutant 3'-UTR (CCNL1-MUT) were transfected into PC-3 and DU145 cells with miR-5195-3p mimics or miR-NC. MiR-5195-3p upregulation suppressed luciferase activity of the wild-type but not the mutant 3'-UTR of CCNL1. Renilla luciferase activity was used as a control. D mRNA and $\mathbf{E}$ protein expression levels of CCNL1 following miR-5195-3p mimics or miR-NC transfection. F CCNL1 mRNA expression levels in 69 pairs of human PCa and matched adjacent normal tissues were measured by quantitative real-time PCR. Data are presented as mean \pm standard deviation. ${ }^{* *} p<0.01,{ }^{* * *} p<0.001$ compared with miR-NC group. G MiR-5195-3p expression was inversely correlated with CCNL1 miRNA expression in PCa tissues, as demonstrated by the Spearman's correlation coefficient 


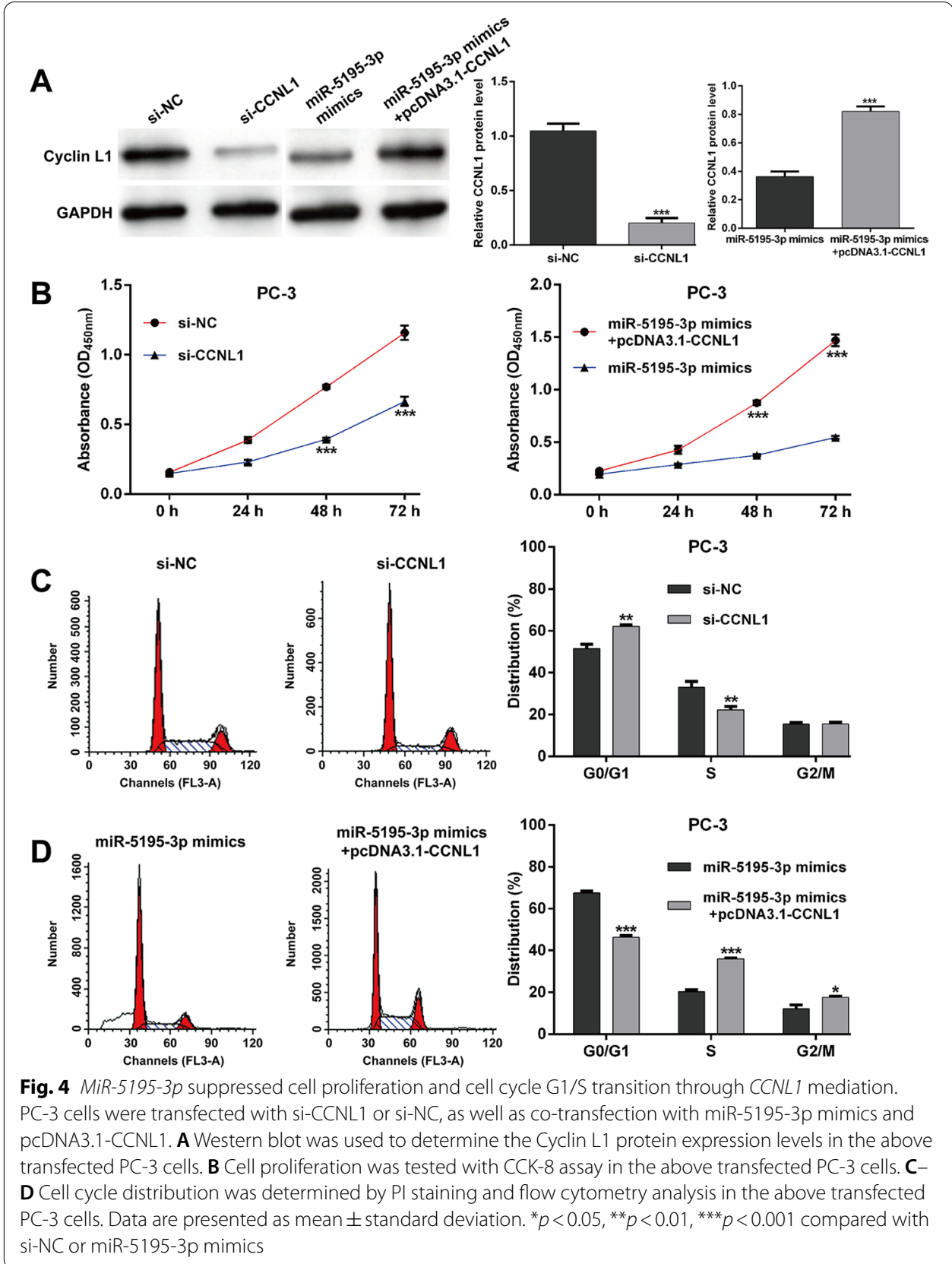

suggest that miR-5195-3p markedly inhibited the proliferation and G1/S transition, at least partially via targeting CCNL1 in PCa.

\section{MiR-5195-3p overexpression restricted tumor growth in vivo}

The xenograft tumorigenicity test was performed to elucidate the in vivo suppressive potential of $m i R-5195-3 p$. Initially, the overexpression of $m i R-5195-3 p$ of $m i R-5195-3 p$ mimics in PC-3 cells was assessed and quantitative real-time PCR findings showed that miR-5195-3p mimics had a high overexpression efficiency (Fig. 5A). Subsequently, stably (miR-5195-3p mimics or miR-NC) transfected PC-3 cells were injected subcutaneously 


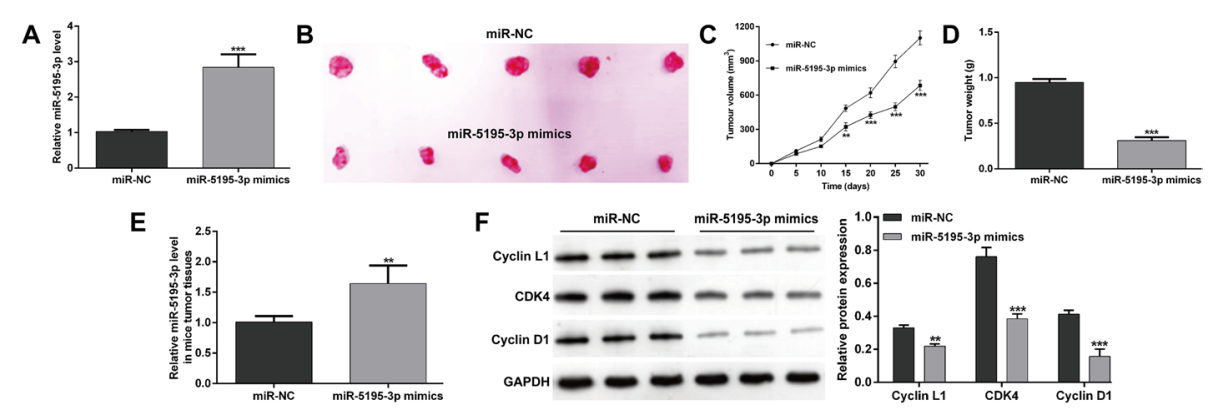

Fig. 5 MiR-5195-3p induced the regression of prostate tumorigenesis in vivo. A MiR-5195-3p expression in stable miR-5195-3p mimics or miR-NC overexpression PC-3 cells determined by quantitative real-time PCR. B Image of tumor xenografts in nude mice injected subcutaneously with miR-5195-3p-overexpressing PC-3 cells. C Tumor volume was measured every 5 days. D Changes in the tumor weight in mice after miR-5195-3p overexpression. E MiR-5195-3p expression in tumor tissue isolated from miR-5195-3p mimics and miR-NC groups of nude BALB/c mice. $\mathbf{F}$ Protein expression levels of Cyclin L1, CDK4, and Cyclin D1 expression were determined by western blot analysis. Data are presented as mean \pm standard deviation. ${ }^{* *} p<0.01$, ${ }^{* * *} p<0.001$ compared with miR-NC

into the right flank of nude mice to produce a xenograft model of human PC-3 tumors. As shown in Fig. 5B, the tumor size was obviously smaller in miR-5195-3p mimics group compared with the miR-NC group in a time course of 30 days. Moreover, the timedependent analysis illustrated that the tumor volume was significantly suppressed in mice inoculated with miR-5195-3p overexpressing PC-3 cells compared with the miRNC group (Fig. 5C). Meanwhile, the tumor weight was also remarkably decreased in miR-5195-3p overexpressed mice (Fig. 5D). Next, we compared the relative expression of $m i R-5195-3 p$ and found a significant high expression of miR-5195-3p expression in $m i R-5195-3 p$ mimics mouse tumor tissue than in miR-NC group mouse tumor tissue (Fig. 5E). We further analyzed the protein levels of Cyclin L1, CDK4, and Cyclin D1 in tumor tissues derived from a subcutaneous xenograft murine model using western blot analysis. As shown in Fig. 5F, the protein expression levels of Cyclin L1, CDK4, and Cyclin D1 were all significantly suppressed in the miR-5195-3p mimics group tumor tissues relative to the miR-NC group mouse tumor tissues, which further confirmed that the upregulation of $m i R-5195-3 p$ inhibited the growth of the prostate tumorigenesis by targeting CCNL1.

\section{Discussion}

The present study revealed that $m i R-5195-3 p$ was significantly downregulated in PCa tissues compared with adjacent normal tissues. Lower abundance of miR-5195-3p was associated with Gleason score, TNM stage, and worse prognosis in patients with PCa, highlighting its potential role as a tumor suppressor miRNA. The in vitro experiments demonstrated that miR-5195-3p overexpression reduced proliferation and induced G0/ G1 cell cycle arrest in PCa cells (PC-3 and DU145). Consistent with our in vitro data, significant reduction in miR-5195-3p was observed in cancer samples, and the reduction was correlated with increased cell proliferation in ovarian cancer [23]. Jiang et al. [17] showed that miR-5195-3p suppressed the proliferation and invasion of human bladder cancer cells. Wang et al. [16] observed a decrease in $m i R-5195-3 p$ expression in osteosarcoma (OS) tissues and further manifested that miR-5195-3p overexpression 
attenuated OS cell proliferation and induced apoptosis. Additionally, miR-5195-3p has been reported to play a suppressive role in cell growth and proliferation in glioma cells [15] and human non-small cell lung cancer cells. In particular, PC-3 and DU145 are two androgen receptor (AR)-negative PCa cell lines presenting relatively lower miR-5195-3p expression in all PCa cell lines, which were thus selected for gain-of-function assays. Based on the in vitro data, miR-5195-3p might play an important role in the development of AR-negative prostate cancer.

Furthermore, we analyzed the effects of miR-5195-3p overexpression on tumorigenesis and found that tumor formation in vivo was reduced with elevated miR-5195-3p abundance, further confirming $m i R-5195-3 p$ functions as a tumor-suppressive miRNA in PCa. At the molecular level, we confirmed the role of $m i R-5195-3 p$ in cell cycle G1/S transition regulation, as reflected by decreased expression of CDK4/Cyclin D1 by miR5195-3 $p$ overexpression in tumor tissues. In fact, uncontrolled proliferation of tumor cells is closely associated with a deregulation of the cell cycle progression directly driven by a series of heterodimers formed by cyclins and cyclin-dependent kinases (CDKs) $[24,25]$. Several lines of evidence indicate that $m i R-5195-3 p$ is an important cell cycle regulator. For instance, $m i R-5195-3 p$ overexpression significantly downregulated the expression levels of c-MYC and cyclin D1 but upregulated p21 expression in HCT116 cells [26]. MiR-5195-3p sharply suppressed the expression of its downstream promoting cell cycle regulator cyclin D1 in bladder cancer cells [17]. Thus, we speculated that miR5195-3p may exert its suppressive effects on PCa cell proliferation via inducing cell cycle G0/G1 arrest through downregulating CDK4/Cyclin D1 expression.

To the best of our knowledge, several target genes of miR-5195-3p, including HOXB6 in hepatocellular carcinoma [27], MYO6 in lung cancer [14], BIRC2 in glioma [15], EIF4A2 in breast cancer [28], NEDD9 in OS [16], and KLF5 in bladder cancer [17], have been identified and confirmed, which were largely associated with aberrantly tumor cell proliferation. Here, we selected CCNL1 as the potential target gene of miR-5195-3p for its role in G1/S transition [18] and carcinogenesis [19]. Furthermore, we demonstrated that $m i R-5195-3 p$ downregulated CCNL1 via directly binding its $3^{\prime}$-UTRs. The expression of miR-5195-3p was inversely correlated with the CCNL1 expression level in 69 paired PCa tissues. Similarly, CCNL1 was reported to be a direct target gene of miR$199 b-5 p$ and be involved in miR-199b-5p suppressing cell proliferation and arresting cell cycle progression in Ewing's sarcoma [22]. As expected, our data illustrated that CCNL1 knockdown imitated the effects of miR-5195-3p overexpression on PCa cell proliferation and cell cycle G1/S transition, while a converse effect was observed with CCNL1 overexpression. Notably, it is interesting that the use of siRNA-mediated depletion of cyclinL1 does not seem to show this increase in apoptotic cells (sub-G1), while miR$5195-3 p$ caused an increase in the proportion of cells at sub-G1 phase in PC-3 cells. In fact, miR-5195-3p induces apoptosis by directly targeting NEDD9 in osteosarcoma [16] and targeting EIF4A2 in breast cancer chemosensitivity [28]. These data suggest that the $m i R-5195-3 p$ has other targets that may be primarily responsible for the apoptosis observed in $\mathrm{PCa}$, which we will investigate in our next work further elucidating the molecular mechanisms underlying miR-5195-3p suppressing PCa cell proliferation. Our work here indicates that CCNL1 is another identified target of miR-5195-3p associated with cell cycle G0/G1 phase arrest induced by miR-5195-3p overexpression in PCa cells. 
We thus speculated that $m i R-5195-3 p$ induced cell cycle G0/G1 arrest by downregulating CDK4/Cyclin D1 via targeting CCNL1. In addition, our study had some limitations: (1) more sample tissues should be collected for analyzing the clinical significance of miR-5195-3p/CCNL1 axis in PCa; (2) more PCa cell lines should be included in Figs. 4 and 5, considering the limited experimental conditions; (3) we did not investigate the effect of miR-5195-3p on AR-positive cell lines; (4) more targets of miR-5195-3p should be identified and validated; (5) other molecular mechanisms underlying the downstream pathway of CCNL1 still need to be further explored.

\section{Conclusions}

In summary, this study established the tumor-suppressive role of miR-5195-3p in PCa in vitro and in vivo. Most importantly, CCNL1 was demonstrated to be the functionally regulated by miR-5195-3p, which was associated with cell cycle G1/S transition. Therefore, $m i R-5195-3 p$ could be a potential diagnostic biomarker and therapeutic target for the treatment and early diagnosis of patients with PCa.

\section{Abbreviations}

PCa: Prostate cancer; CCNL 1: Cyclin L1; SDS-PAGE: Sodium dodecyl sulfate polyacrylamide gel electrophoresis; HR: Hazard ratio.

Acknowledgements

Not applicable.

\section{Authors' contributions}

ZX and HZQ performed the experiments and collected the original data. SYQ analyzed the data. WX and GJH participated in the interpretation of data and drafted the manuscript. LZ designed the experiments, contributed reagents or materials, authored or reviewed drafts of the paper, and approved the final draft. All authors have read and approved the manuscript.

\section{Funding}

No applicable.

Availability of data and materials

The dataset supporting the conclusions of this article is included within the article.

\section{Declarations}

\section{Ethics approval and consent to participate}

All tissue samples were obtained in accordance with the Declaration of Helsinki (1975) and approved by the ethics committee of Tongji Hospital (approval number TMCU-87DG; 2016.3.12; Hubei, China). All animal experiments were performed under the approval of in accordance with the Huazhong University of Science and Technology Research Institute Animal Care Committee guidelines (approval number HUST-58A; 2017.6.23; Hubei, China).

\section{Consent for publication}

Not applicable.

\section{Competing interests}

The authors declare that they have no competing interests.

Received: 14 December 2021 Accepted: 22 February 2022

Published online: 08 March 2022

\section{References}

1. Ferlay J, Soerjomataram I, Dikshit R, Eser S, Mathers C, Rebelo M, et al. Cancer incidence and mortality worldwide: sources, methods and major patterns in GLOBOCAN 2012. Int J Cancer. 2015;136(5):E359-86.

2. Miller KD, Siegel RL, Lin CC, Mariotto AB, Kramer JL, Rowland JH, et al. Cancer treatment and survivorship statistics, 2016. CA Cancer J Clin. 2016;66(4):271-89.

3. Siegel RL, Miller KD, Jemal A. Cancer statistics, 2019. CA Cancer J Clin. 2019;69(1):7-34.

4. Shao N, Wang Y, Jiang WY, Qiao D, Zhang SG, Wu Y, et al. Immunotherapy and endothelin receptor antagonists for treatment of castration-resistant prostate cancer. Int J Cancer. 2013;133(7):1743-50. 
5. Wong YN, Ferraldeschi R, Attard G, de Bono J. Evolution of androgen receptor targeted therapy for advanced prostate cancer. Nat Rev Clin Oncol. 2014;11(6):365-76.

6. Nevedomskaya E, Baumgart SJ, Haendler B. Recent advances in prostate cancer treatment and drug discovery. Int J Mol Sci. 2018;19(5):1359.

7. Almeida MI, Reis RM, Calin GA. MicroRNA history: discovery, recent applications, and next frontiers. Mutat Res. 2011;717(1-2):1-8.

8. Filipowicz W, Bhattacharyya SN, Sonenberg N. Mechanisms of post-transcriptional regulation by microRNAs: are the answers in sight? Nat Rev Genet. 2008;9(2):102-14.

9. Nam RK, Benatar T, Wallis CJD, Kobylecky E, Amemiya Y, Sherman C, et al. MicroRNA-139 is a predictor of prostate cancer recurrence and inhibits growth and migration of prostate cancer cells through cell cycle arrest and targeting IGF1R and AXL. Prostate. 2019;79(12):1422-38.

10. Ji L, Jiang X, Mao F, Tang Z, Zhong B. miR-589-5p is downregulated in prostate cancer and regulates tumor cell viability and metastasis by targeting CCL-5. Mol Med Rep. 2019;20(2):1373-82.

11. Zhang Y, Zhang D, LV J, Wang S, Zhang Q. miR-410-3p promotes prostate cancer progression via regulating PTEN/ AKT/mTOR signaling pathway. Biochem Biophys Res Commun. 2018;503(4):2459-65.

12. Bi CW, Zhang GY, Bai Y, Zhao B, Yang H. Increased expression of miR-153 predicts poor prognosis for patients with prostate cancer. Medicine. 2019;98(36):e16705.

13. Liu JB, Yan YJ, Shi J, Wu YB, Li YF, Dai LF, et al. Upregulation of microRNA-191 can serve as an independent prognostic marker for poor survival in prostate cancer. Medicine. 2019;98(29):e16193.

14. Yang Q. MicroRNA-5195-3p plays a suppressive role in cell proliferation, migration and invasion by targeting MYO6 in human non-small cell lung cancer. Biosci Biotechnol Biochem. 2019;83(2):212-20.

15. Yang J, Yan DM, Xhu LX, Si DM, Liang QH. MiR-5195-3p inhibits the proliferation of glioma cells by targeting BIRC2. Eur Rev Med Pharmacol Sci. 2020;24(1):267-73.

16. Wang L, Shi G, Zhu D, Jin Y, Yang X. miR-5195-3p suppresses cell proliferation and induces apoptosis by directly targeting NEDD9 in osteosarcoma. Cancer Biother Radiopharm. 2019;34(6):405-12.

17. Jiang Z, Zhang Y, Cao R, Li L, Zhong K, Chen Q, et al. miR-5195-3p inhibits proliferation and invasion of human bladder cancer cells by directly targeting oncogene KLF5. Oncol Res. 2017;25(7):1081-7.

18. Berke JD, Sgambato V, Zhu PP, Lavoie B, Vincent M, Krause M, et al. Dopamine and glutamate induce distinct striatal splice forms of Ania-6, an RNA polymerase II-associated cyclin. Neuron. 2001;32(2):277-87.

19. Mitra S, Mazumder Indra D, Basu PS, Mondal RK, Roy A, Roychoudhury S, et al. Amplification of cyclinL1 in uterine cervical carcinoma has prognostic implications. Mol Carcinog. 2010;49(11):935-43.

20. Redon R, Hussenet T, Bour G, Caulee K, Jost B, Muller D, et al. Amplicon mapping and transcriptional analysis pinpoint cyclin L as a candidate oncogene in head and neck cancer. Cancer Res. 2002;62(21):6211-7.

21. Sticht C, Hofele C, Flechtenmacher C, Bosch FX, Freier K, Lichter P, et al. Amplification of Cyclin L1 is associated with lymph node metastases in head and neck squamous cell carcinoma (HNSCC). Br J Cancer. 2005;92(4):770-4.

22. LiW, LiY, Guo J, Pan H, Zhang Y, Wang X. Overexpression of miR-199b-5p inhibits Ewing's sarcoma cell lines by targeting CCNL1. Mol Med Rep. 2015;12(3):3359-64.

23. Ebrahimi SO, Reiisi S. Downregulation of miR-4443 and miR-5195-3p in ovarian cancer tissue contributes to metastasis and tumorigenesis. Arch Gynecol Obstet. 2019;299(5):1453-8.

24. Tenga MJ, Lazar IM. Proteomic snapshot of breast cancer cell cycle: G1/S transition point. Proteomics. 2013;13(1):48-60.

25. Malumbres M, Barbacid M. Cell cycle, CDKs and cancer: a changing paradigm. Nat Rev Cancer. 2009:9(3):153-66.

26. Jahangiri Moez M, Bjeije H, Soltani BM. Hsa-miR-5195-3P induces downregulation of TGF $\beta R 1, T G F \beta R 2$, SMAD3 and SMAD4 supporting its tumor suppressive activity in HCT116 cells. Int J Biochem Cell Biol. 2019:109:1-7.

27. Li Y, Jiang A. ST8SIA6-AS1 promotes hepatocellular carcinoma by absorbing miR-5195-3p to regulate HOXB6. Cancer Biol Ther. 2020;21(7):647-55.

28. Liu M, Gong C, Xu R, Chen Y, Wang X. MicroRNA-5195-3p enhances the chemosensitivity of triple-negative breast cancer to paclitaxel by downregulating EIF4A2. Cell Mol Biol Lett. 2019;24:47.

\section{Publisher's Note}

Springer Nature remains neutral with regard to jurisdictional claims in published maps and institutional affiliations.

Ready to submit your research? Choose BMC and benefit from:

- fast, convenient online submission

- thorough peer review by experienced researchers in your field

- rapid publication on acceptance

- support for research data, including large and complex data types

- gold Open Access which fosters wider collaboration and increased citations

- maximum visibility for your research: over $100 \mathrm{M}$ website views per year

At BMC, research is always in progress.

Learn more biomedcentral.com/submissions 